Review Article

\title{
Novel Nanomaterials Enable Biomimetic Models of the Tumor Microenvironment
}

\author{
Marshall Hunter Joyce, Shane Allen, Laura Suggs, and Amy Brock \\ Department of Biomedical Engineering, The University of Texas at Austin, Austin, TX 78712, USA \\ Correspondence should be addressed to Amy Brock; amy.brock@austin.utexas.edu
}

Received 17 March 2017; Revised 19 June 2017; Accepted 22 August 2017; Published 12 October 2017

Academic Editor: Yi Zhang

Copyright (c 2017 Marshall Hunter Joyce et al. This is an open access article distributed under the Creative Commons Attribution License, which permits unrestricted use, distribution, and reproduction in any medium, provided the original work is properly cited.

In the complex tumor microenvironment, chemical and mechanical signals from tumor cells, stromal cells, and the surrounding extracellular matrix influence all aspects of disease progression and response to treatment. Modeling the physical properties of the tumor microenvironment has been a significant effort in the biomaterials field. One challenge has been the difficulty in altering the mechanical properties of the extracellular matrix without simultaneously impacting other factors that influence cell behavior. The development of novel materials based on nanotechnology has enabled recent innovations in tumor cell culture models. Here, we review the various approaches by which the tumor cell microenvironment has been engineered using natural and synthetic gels. We describe new studies that rely on the unique temporal and spatial control afforded by nanomaterials to produce culture platforms that mimic dynamic changes in tumor matrix mechanics. In addition, we look at the frontier of nanomaterial-hydrogel composites to review new approaches for perturbation of mechanochemical control in the tumor microenvironment.

\section{Introduction}

Cancer is a complex disease with numerous components that are proving more relevant to disease progression each year. A major difficulty when studying any disease is constructing a model that is not only easy to work with, but one that provides reliable results that can be translated to clinical use. Nanoparticles have proven capable of simulating key aspects of tumorigenesis and tumor progression, especially those aspects relating to the cellular environment in which the tumor exists, termed the "tumor microenvironment."

\section{Microenvironmental Control of Tumorigenesis: A Role for Matrix Mechanics}

Cancer is a disease of the entire tissue and is characterized by alterations in the cellular phenotypes and composition as well as changes in the noncellular proteins, glycoproteins, and proteoglycans that comprise the extracellular matrix (ECM). Major components of the ECM include fibrillar collagen, as well as basement membrane proteins such as type IV collagen, laminin, and fibronectin. These play major roles as structural proteins as well as functional regulators of signaling pathways. The interplay between tumor cells, stromal cells, and ECM contributes to the formation of a microenvironment that determines the tumor outcome.

Interactions between cells and ECM are mediated by a family of dimeric integrin receptors. These transmembrane receptors bind to ECM fibrils on the cell surface and attach to intracellular actin microfilaments through linker focal adhesion proteins. Integrin-mediated cell adhesions are critical in sensing and transmitting myosin-based cytoskeletal contractile forces and external forces from the matrix environment. Mechanical forces activate a wide range of signaling pathways in physiological cell function and disease.

A landmark study in 1997 demonstrated that integrinmediated adhesions with the surrounding ECM drive the expression of malignant phenotypes [1-3]. This work relied on the three-dimensional (3D) culture of mammary epithelial cells embedded in the ECM. While epithelial cells grow in monolayers on two-dimensional culture dishes, $3 \mathrm{D}$ culture in the ECM induces the growth of spheroid structures mimicking in vivo acinar organization. Nonmalignant epithelial cells establish hollow spheroids with a central 


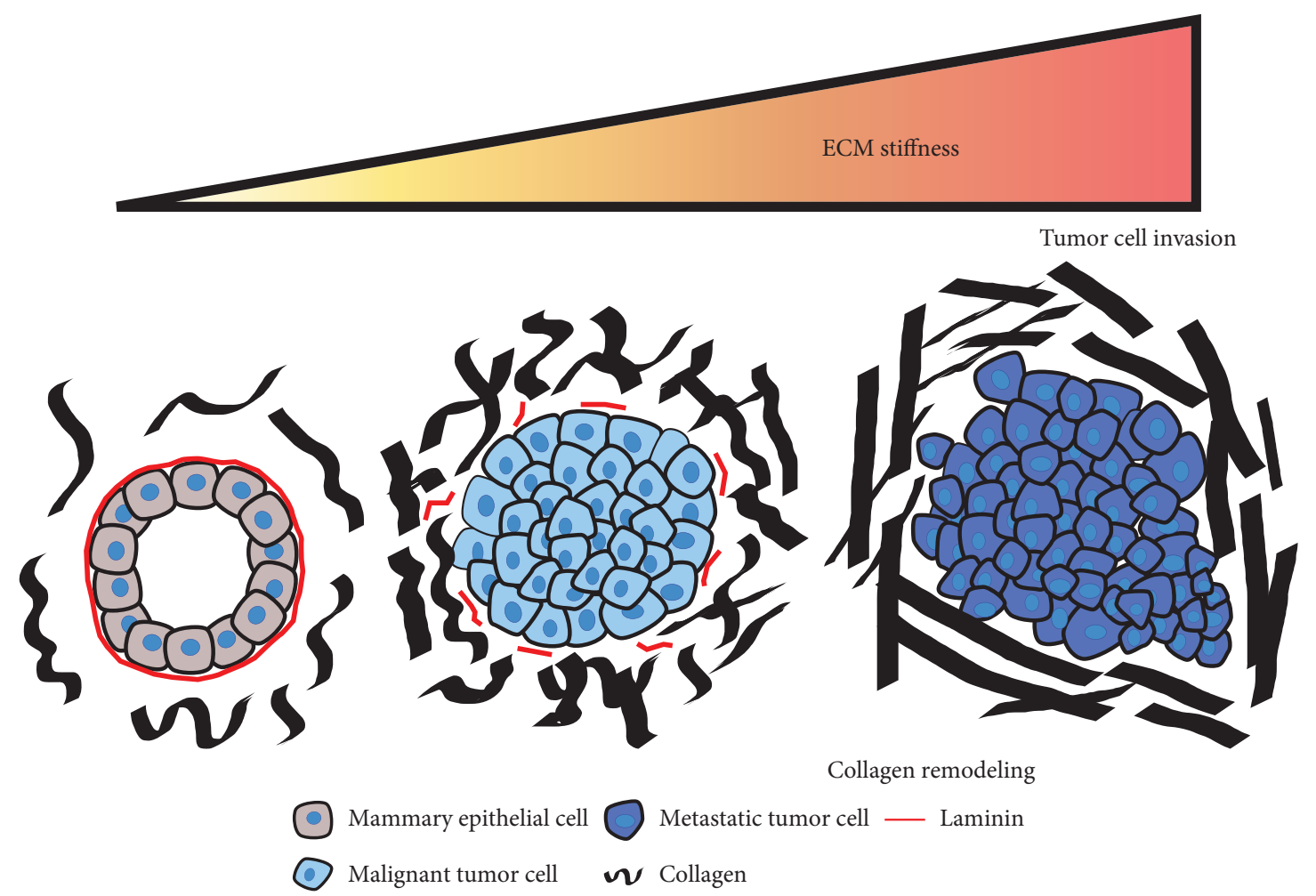

(a)

Nonmalignant

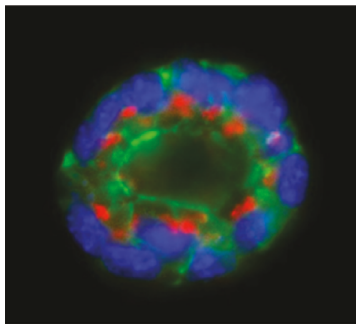

(b)

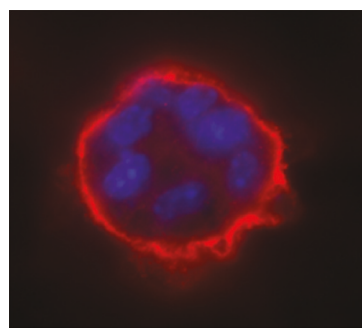

(e)
Malignant

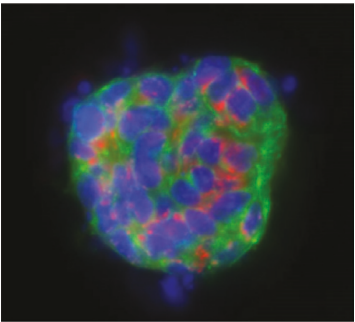

(c)

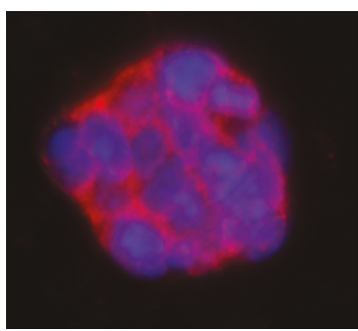

(f)
Metastatic

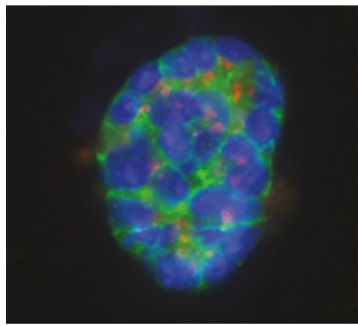

(d)

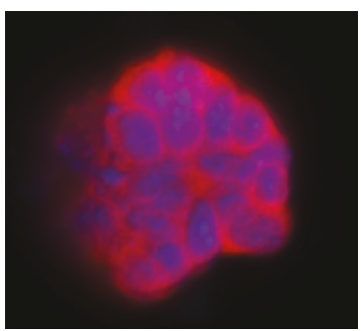

(g)

Figure 1: Mechanical cues from the extracellular matrix help drive tumorigenesis. (a) Increasing collagen production and cross-linking causes a stiffening of the extracellular matrix (ECM). This increasing mechanical load on the microenvironment has been shown to alter phenotypic expression of cells towards malignant phenotypes. (b, e) Nonmalignant mammary epithelial cells will form spherical acini with apical/basal polarity, a basement membrane, and a hollow lumen. (c, f) The organized structure is lost in malignant cells, as is the hollow lumen. (d, g) Metastatic cells exhibit similar phenotypes to malignant cells with the loss of apical/basal polarity, basement membrane, and hollow lumen. For images (b-d), mammary spheroids are stained for GM130 (red), DAPI (blue), and actin (green). Images (e, f) show mammary spheroids stained for laminin (red) and DAPI (blue).

hollow lumen, polarize apical and basal surfaces, and deposit laminin and collagen IV basement membrane (Figures 1(a), 1(b), and 1(e)). In contrast, tumorigenic cells form spheroids without a hollow lumen and remain poorly organized with diffuse expression of apical- and basalspecific proteins (Figures 1(a), 1(c), 1(d), 1(f), and 1(g)). Weaver et al. showed that treatment with $\beta 1$-integrininhibiting antibodies was sufficient to induce tumorigenic 
cells to grow as morphological normal epithelial spheroids. Through continuous culture with/without the $\beta 1$-integrininhibiting antibodies, it was also clear that the switch between tumorigenic and nonmalignant phenotype was reversible. This study established that integrin-mediated interaction between cells and ECM impacts the expression of whole programs of gene expression, producing genome-wide changes associated with phenotype switching [1].

Similar results have linked expression of alpha(v)beta(3) integrin (Int- $\alpha v \beta 3$ ) to expression of malignant phenotypes in MCF7 ER ${ }^{+}$breast carcinoma cells [4]. Abu-Tayeh et al. used basement membrane extract to generate 3D cultures of MCF7 cells and were able to measure malignancy based on acinar formation, apical-basal polarity, and presence/absence of a hollow lumen. Examination of paraffin-embedded breast tissues indicated that Int- $\beta 3$ expression is lost in the early stages of tumorigenesis, but MCF7 clones engineered to reexpress Int- $\beta 3$ were capable of forming acinar-like structures similar to "normal" mammary tissue when cultured in basement membrane extract. Treatment with cilengitide, an agent that blocks Int- $\alpha v \beta 3$ and Int- $\alpha v \beta 5$ activity, decreased acinar-like structure formation. Heparan sulfate proteoglycans (HSPGs), another mechanotransduction-associated surface protein, have been found to mediate progression of prostate cancer cells through modulation of production of ECMdegrading enzymes called matrix metalloproteinases (MMPs) [5]. Elevated MMP expression correlates to increased metastatic potential in numerous types of cancer; one hypothesis suggests that tumor cells adjust their MMP expression to increase the pore size of their ECM, allowing the cells to migrate easier and thereby facilitating tumor progression [6-9]. Pancreatic cancer cells (Panc-1) cultured on collagen hydrogels of increasing stiffness were shown to increase MMP activity [5]. Glutaraldehyde and transglutaminase were used to cross-link the collagen hydrogels, and contractility inhibitors (Y-27632, ML-7, and blebbistatin) were added in order to isolate the effect of substrate stiffness alone. These studies demonstrate that ECM stiffness causes increased activity of MMP in some cancer cells and is another mechanism of tumor progression.

The ECM has also been shown to facilitate the invasion of tumor cells that have been activated by an oncogene [10]. Mammary epithelial cells (MCF10A) were cultured in collagen $\mathrm{I} /$ reconstituted basement membrane $3 \mathrm{D}$ cultures and formed acini with a hollow lumen and apical-basal polarity, indicative of a "normal" phenotype. Activation of oncogenic ErbB2 signaling in control cultures caused luminal filling and destabilization of cell-cell junctions, but apical-basal polarity was retained and cells were noninvasive. However, in the context of stiff ECM, activation of the same pathway resulted in loss of apical-basal polarity and invasion into the gels. This study provides direct evidence that physical cues from a cell's microenvironment can direct tumorigenesis and may even exert a dominant effect over oncogenic mutations.

The effects of ECM stiffness have been shown to promote tumor progress independent of composition or architecture. Studies where nonmalignant mammary epithelial cells (MCF10A) were cultured on Matrigel-alginate hydrogels demonstrated that altering the stiffness of the ECM alone was sufficient to increase Akt phosphorylation, a downstream effector of the PI3K pathway which is often activated in breast cancer and known to drive proliferation and migration [11, 12]. Similar studies in collagen hydrogels demonstrated that increased ECM stiffness leads to increased cell-matrix adhesions through focal adhesion kinases (FAKs) and induction of malignant phenotypes in mammary epithelial cells via Rho-ERK pathways $[13,14]$.

\section{Experimental Models of the Matrix Microenvironment}

To address the complex series of issues in modeling the tumor microenvironment, it is necessary to move beyond standard 2D cell culture models [15]. Specifically in cancer, it has been shown that cells grown within 3D hydrogel substrates influence development of cell aggregates and chemotherapeutic resistance $[16,17]$. Evaluating the role of matrix stiffening on tumor progression, malignant transformation, and therapeutic response has been experimentally challenging due to some remaining limitations of in vitro cell model systems.

One challenge has been the development of matrices that scale with physiologically relevant stiffness alterations. Many studies have been performed in matrices that are initially much stiffer than normal, healthy tissue. Cells cultured in these excessively stiff environments never adopt native morphologies. To overcome these limitations, matrices that can be stiffened over time are needed to mimic the invasive transformations that occur during early stages of cancer. Another challenge relates to the time scale of mechanical alterations in the tissue environment. Even at the early stages of hyperplasia and atypia, precancerous lesions are stiffer than surrounding healthy tissue [18], and this stiffness increases as tumors progress $[10,19,20]$. A causal link between increased matrix stiffness and mammary tumorigenesis has been provided, with experiments composed of mammary epithelial cells (MCF10A) cultured in high- or low-density collagen matrices [21]. The studies indicated that cells cultured in high-density matrices had triple the proliferation rate and distinctly decreased acini organization compared to their low-density matrix counterparts. Mouse models (PyVT/Colla1 $\left.{ }^{\text {tmJae }}\right)$ designed to exhibit both collagen-dense mammary tissue and mammary tumorigenesis showed a threefold increase in tumor formation by 8 weeks compared to control groups. The collagen-dense groups also showed evidence of tumor invasion in regions with increased frequency of reorganized collagen fibers, suggesting that denser tissues had organized ECM components in such a way that aided tumor cell invasion.

\section{Cell Models for Static Control of Matrix Mechanics}

Hydrogels used for breast tumor-derived cell culture have historically used nonsynthetic platforms such as Matrigel, collagen, or other purified basement membrane proteins as they occur naturally within the developing tumor microenvironment and provide a similar environment to the in 
vivo tumor [22-24]. While culture in these matrices more accurately reproduces in vivo cell behavior compared with 2D monolayer culture, the protocols are generally more complex and labor-intensive. Batch-to-batch variations in the ECM are a concern [24], and the use of Matrigel may not be appropriate for applications in which precise knowledge of the matrix composition is required. To address these limitations, bioinert polymer-based matrices have been functionalized with cell adhesive ligands to generate culture substrates that are well defined [17, 25, 26].

A common strategy has been to construct polyacrylamide (PA) gels, whose elastic modulus can be easily controlled by varying cross-linker concentration. Cell attachment is facilitated by coating the PA gel surface with cell adhesive ligands [27]. PA hydrogel surfaces have been utilized to investigate the role of mechanical substrate stiffness on cell morphology, cytoskeletal structure, stem cell differentiation, and tumor progression [28, 29]. MCF10A murine epithelial cells undergo TWIST-dependent epithelial to mesenchymal transition (EMT) from a normal mammary phenotype to an invasive one when cultured on stiff $(6 \mathrm{kPa})$ PA substrates [30]. MCF10A cultured on stiffer substrates produce increased levels of fibronectin as well [31]. To study the role of substrate stiffness in cell response to the chemotherapeutic drug paclitaxel, a panel of cancer cell types was cultured on $100 \mathrm{kPa}$ and $1 \mathrm{kPa} \mathrm{PA}$ gel substrates [32]. Cervical adenocarcinoma (HeLa) and neuroblastoma (SY5Y) cells demonstrated stiffness-dependent paclitaxel resistance, with IC50 doses shifted significantly higher in the cells cultured on stiffer substrates [32].

While these coated PA gel surfaces provide highthroughput models to investigate cell behavior, they do not recapitulate the $3 \mathrm{D}$ structures produced by similar cell types in vivo. To more accurately model the mechanics of the tumor environment, it is necessary to assess the behavior of cells embedded within a matrix. The Weaver group encapsulated murine epithelial cells within collagen alone or combined with other basement membrane matrices; stiffness could be altered by modulating initial collagen concentration [14]. Cells cultured in softer $(4 \mathrm{kPa})$ substrates developed similarly to normal mammary tissue; however, culture in the stiffer $(150 \mathrm{kPa})$ substrate leads to loss of normal apical-basal polarity and increased growth. These phenotypes relied on focal adhesions as the key force transducer connecting the cellular cytoskeleton with the matrix environment [14]. This malignant transformation was further described through a series of hydrogels of increasing elastic moduli between 100 and $1000 \mathrm{~Pa}$. As the stiffness of their substrate increased, MCF10A cell aggregates lost apical-basal polarity and grew to increasingly larger sizes; this mechanism required PI3K and Racl mechanotransduction pathways and was abrogated through an increase in laminin content [11]. This experimental system was additionally used to characterize microRNA expressiondriven integrin activation as a driver of stiffness-dependent cell phenotype transformation [33] and to assess variation in in vitro chemotherapeutic resistance in multiple tumor lines $[17,32]$. Chronic myeloid leukemia cells varied in growth dynamics and drug sensitivity based upon the rigidity of their microenvironment [17]. This research highlights the need for increased attention to microenvironment control parameters in preclinical testing of chemotherapeutics.

Models utilizing hydrogels with static mechanical properties have shown great utility in investigation of carcinogenesis in vitro, but they fail to capture the dynamic nature of the tumor microenvironment. Tumors increase in stiffness and simultaneously reorganize their surrounding matrix throughout disease progression [10, 34] (Figure 1). It is therefore necessary to incorporate cell culture platforms with dynamic capabilities to capture shifts in tumor mechanics.

\section{Nanomaterial-Based Dynamic Models of the Tumor Microenvironment}

The tumor microenvironment exhibits alterations in bulk modulus, ECM structure and composition, and stromal cell composition throughout the course of disease progression by changing the concentration of the structural protein or polysaccharide $[10,34,35]$. Cells respond to both increasing cross-linking density and concentration, making it difficult to resolve the contribution of each component. Tuning the matrix stiffness after gel formation adds another level of engineering challenge, but it is imperative to study natural processes that occur in dynamic matrices during disease progression. Here, we will focus on recent efforts to integrate dynamic changes in the mechanics of the tumor microenvironment into new model systems for in vitro study.

Synthetic poly(ethylene glycol) (PEG) hydrogels are utilized heavily in this field due to the tight control of their properties and relative simplicity in the addition of functionality through a variety of chemistries; popular additions of matrix metalloproteinase (MMP) sensitive, cell adhesive, or cross-linking are achieved through "click" chemistry $[36,37]$. Mechanical properties can be modified by incorporating photoinitiators, such that irradiation at specific wavelengths induces additional cross-linking of the gel backbone and thus increases the bulk gel elastic modulus [38]. This functionality is not limited to PEG-based hydrogels; a similar scheme of cross-linking methacrylated hyaluronic acid (HA) has been used to stiffen gels from 3 to $30 \mathrm{kPa}$ and to investigate mesenchymal stem cell differentiation [39]. Similar systems have been developed to reduce the modulus of hydrogel models. The Anseth group developed a PEG hydrogel, incorporating a photocleavable nitrobenzyl ether moiety within the backbone. Upon exposure to UV light, backbone cross-links are cleaved, thereby reducing the elastic modulus of the hydrogel. A similar strategy can incorporate the photocleavable moiety adjacent to cell adhesive ligands to achieve tight control over matrix chemical composition [26].

While UV-sensitive cross-linkers can be used to generate hydrogels with dynamic-stiffening capability, a caveat is that the cross-linking agents remain in the gel throughout the study and alter the backbone of the hydrogel itself. A remarkably clever alternative avoids the presence of a photoinitiator or other chemical compounds by modulating the conformation of an azobenzene in a PEG hydrogel backbone. The azobenzene moiety converts to a less rigid 


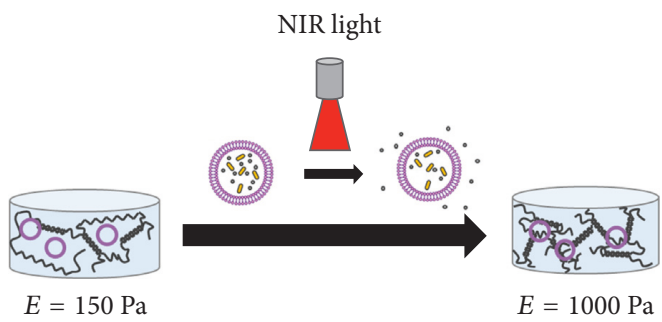

(a)
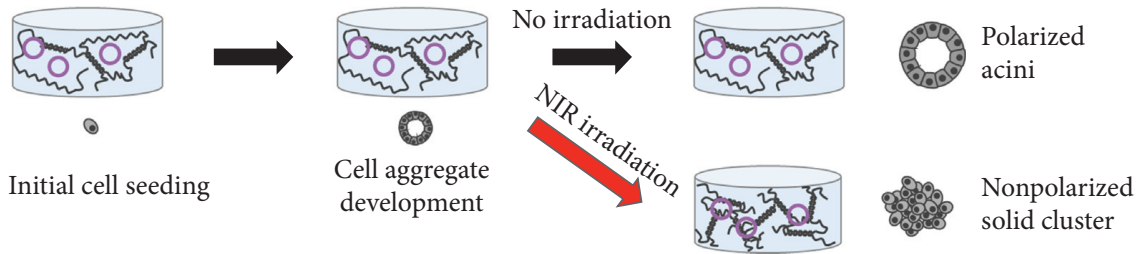

(b)

FIgURE 2: Epithelial cell transformation in dynamic culture system. (a) Alginate-Matrigel IPN loaded with liposomes containing calcium and AuNR. Calcium is released from the liposomes via AuNR SPR upon NIR irradiation, stiffening the bulk elastic modulus (E) from 150 to $1000 \mathrm{~Pa}$. (b) Mammary epithelial cells encapsulated within the soft gel for polarized cell aggregates (acini) over culture. However, stiffening of this gel disrupts acinar formation, leading to invasive clusters.

cis isomer with UV light and converts back to trans state with removal of UV or addition of visible light. As a result, the hydrogel can be reversibly weakened by up to $1000 \mathrm{~Pa}$ with the addition of UV light [40]. Another method utilizes liposomes loaded with calcium ions and gold nanorods (AuNRs) distributed within an alginate-Matrigel interpenetrating network (IPN) hydrogel (Figure 2). The nanomaterial gels at with an initial defined stiffness; however, upon near-infrared irradiation, the AuNRs in the liposomes undergo surface plasmon resonance, producing heat which permeabilizes the lipid membrane and triggers release of calcium ions. The increase in calcium concentration results in alginate cross-linking, effectively increasing the stiffness of the gel from 150 to $1000 \mathrm{~Pa}$ (Figure 2(a)). This process can also operate in the reverse direction, with a calcium chelator loaded in place of the $\mathrm{CaCl}_{2}$; upon release, calcium is chelated from the alginate, reducing the stiffness of the gel [41]. This model has been used to investigate MCF10A malignant transformation in vitro and found that dynamic stiffening of the matrix promoted a phenotypic change whereby cell aggregates lost polarity and developed invasive fronts [42] (Figure 2(b)).

\section{Nanoparticle-Hydrogel Composites}

A major application of nanoparticle-hydrogel composites in tumor biology has been in the form of injectable materials for in vivo investigation. While not specifically targeting the mechanical aspect of the tumor microenvironment, these do act on other factors relevant to therapy and the history of nanomaterials in the context of tumor biology. A large number of these further compartmentalize in the form of therapeutic delivery. Hydrogels composed in part of nanoparticles have been used for many years as controllable drug delivery, and many use light-triggered hydrogel collapse to release drugs [43]. The most prominent family of nanoparticles used in conjunction with hydrogels are goldand silica-based nanoparticles. Gold nanoparticles (AuNPs) fall into three major families: nanospheres, nanoshells, and nanorods; by altering the shape and content of the nanoparticle, the surface plasmon resonance (SPR) wavelength can be altered [44]. Upon irradiation of these nanoparticles at their SPR wavelength, the nanoparticle absorbance cross section increases resulting in the alteration of many properties, with an increase in local heating most utilized for biologic systems [45].

Applications of these composite hydrogels rely on the increase in local heating to alter the properties of the hydrogel for drug delivery applications. For example, one group synthesized a poly( $N$-isopropylacrylamide)- (pNIPAAm-) based hydrogel with lower critical solution temperature (LCST) above physiologic temperature, and thus, the gel swells when in the body. Gold nanoshells are incorporated within the hydrogel, along with doxorubicin (DOX), such that near-infrared (NIR) irradiation heats up the gel past the LCST, collapsing it and releasing the DOX [46]. This strategy has been used with other styles of gold nanoparticles to the same end [47]. Recently, more complex hydrogel composites are becoming common. A hydrogel with a DNA backbone permitted tethering of DNA-functionalized AuNP via complementary base pair binding; upon irradiation at the AuNP SPR, the DNA cross-link melts releasing the AuNP and any encapsulated drug [48]. Additional composite hydrogels have been similarly created to be sensitive to $\mathrm{pH}$, which is particularly important within the tumor microenvironment [49].

One particularly interesting take on composite hydrogel models of drug delivery created a hydrogel with DNAfunctionalized AuNP as a cross-linker. In addition to the therapeutic, the gel is loaded with liposomes with another 
DNA moiety and calcein. The gel is injected with free calcein and gels initially; however, as free calcein diffuses out, bursting the liposomes due to osmotic gradient, the released DNA preferentially binds to the nanoparticle breaking the cross-link releasing the drug [50]. While the majority of these composite hydrogels for this purpose deliver traditional chemotherapeutics, such as DOX, there have been pushes to incorporate a more novel therapeutic, such as siRNA [51]. Additionally, while typically NIR or other light is typically used to release therapeutic, other methods are available, such as external magnetic field application [52].

While a large portion of composite hydrogel development in cancer biology is focused on drug delivery, there are some systems which aim at modeling the mechanic environment or detection of tumor cells. For example, Ayyub and Kofinas synthesized a composite poly(ethylene glycol) (PEG) hydrogel containing enzymatically degradable cross-links and silica nanoparticles within. As the cross-links are cleaved, moieties on the gel backbone are exposed which adsorb to the surface of the nanoparticle, dynamically stiffening the gel from 70 to $850 \mathrm{~Pa}$ [53]. Another group utilized a composite AuNP, silica, and graphene oxide surface as an electrochemical sensor to detect tumor cells through $\mathrm{H}_{2} \mathrm{O}_{2}$ release [54].

\section{Conclusion}

Landmark studies from recent years have demonstrated that the tumor microenvironment's role in cancer is too big to ignore. With growing interest in the development of more accurate biomimetic in vitro models of tumors, novel nanomaterials can overcome current limitations in recapitulating the dynamic tumor microenvironment. These tunable, responsive materials may provide new options for matching both the composition and the mechanical properties of the matrix microenvironment in a patient-specific manner. Furthermore, they offer the capacity to control the temporal evolution of the tumor microenvironment to reflect the time course of dynamic changes associated with disease progression or response to treatment. Nanomaterials are helping to recapitulate key aspects of the disease model and, with continued research, could continue to assist in the construction of in vitro models that consistently produce results that can be translated to the clinic.

\section{Conflicts of Interest}

The authors declare that they have no conflicts of interest.

\section{Acknowledgments}

The authors are grateful to Amy Price for administrative support. This work was supported by Texas 4000 Foundation (to Amy Brock) and the Cancer Prevention \& Research Institute of Texas (RP130372 to Laura Suggs).

\section{References}

[1] V. M. Weaver, O. W. Petersen, F. Wang et al., "Reversion of the malignant phenotype of human breast cells in three-dimensional culture and in vivo by integrin blocking antibodies," Journal of Cell Biology, vol. 137, no. 1, pp. 231-245, 1997.

[2] P. Briand, O. W. Petersen, and B. van Deurs, "A new diploid nontumorigenic human breast epithelial cell line isolated and propagated in chemically defined medium," In Vitro Cellular \& Developmental Biology, vol. 23, no. 3, pp. 181-188, 1987.

[3] K. V. Nielsen and P. Briand, "Cytogenic analysis of in vitro karyotype evolution in a cell line established from nonmalignant human mammary epithelium," Cancer Genetics and Cytogenetics, vol. 39, no. 1, pp. 103-118, 1989.

[4] H. Abu-Tayeh, K. Weidenfeld, A. Zhilin-Roth et al., "Normalizing' the malignant phenotype of luminal breast cancer cells via alpha(v)beta(3)-integrin," Cell Death and Disease, vol. 7, no. 12, p. e2491, 2016.

[5] A. Haage and I. C. Schneider, "Cellular contractility and extracellular matrix stiffness regulate matrix metalloproteinase activity in pancreatic cancer cells," FASEB Journal, vol. 28, no. 8, pp. 3589-3599, 2014.

[6] E. A. Garbett, M. W. Reed, and N. J. Brown, "Proteolysis in human breast and colorectal cancer," British Journal of Cancer, vol. 81, no. 2, pp. 287-293, 1999.

[7] M. Balduyck, F. Zerimech, V. Gouyer et al., "Specific expression of matrix metalloproteinases 1, 3, 9, and 13 associated with invasiveness of breast cancer cells in vitro," Clinical \& Experimental Metastasis, vol. 18, no. 2, pp. 171-178, 2000.

[8] D. Ohlund, B. Ardnor, M. Oman, P. Naredi, and M. Sund, "Expression pattern and circulating levels of endostatin in patients with pancreas cancer," International Journal of Cancer, vol. 122, no. 12, pp. 2805-2810, 2008.

[9] M. Määttä, Y. Soini, A. Liakka, and H. Autio-Harmainen, "Differential expression of matrix metalloproteinase (MMP)2, MMP-9, and membrane type 1-MMP in hepatocellular and pancreatic adenocarcinoma: implication for tumor progression and clinical prognosis," Clinical Cancer Research, vol. 6, no. 7, pp. 2726-2734, 2000.

[10] K. R. Levental, H. Yu, L. Kass et al., "Matrix crosslinking forces tumor progression by enhancing integrin signaling," Cell, vol. 139, no. 5, pp. 891-906, 2009.

[11] O. Chaudhuri, S. T. Koshy, C. B. da Cunha et al., "Extracellular matrix stiffness and composition jointly regulate the induction of malignant phenotypes in mammary epithelium," Nature Materials, vol. 13, no. 10, pp. 970-978, 2014.

[12] T. L. Yuan and L. C. Cantley, "PI3K pathway alterations in cancer: variations on a theme," Oncogene, vol. 27, no. 41, pp. 5497-5510, 2008.

[13] P. P. Provenzano, D. R. Inman, K. W. Eliceiri, and P. J. Keely, "Matrix density-induced mechanoregulation of breast cell phenotype, signaling and gene expression through a FAK-ERK linkage," Oncogene, vol. 28, no. 49, pp. 4326-4343, 2009.

[14] M. J. Paszek, N. Zahir, K. R. Johnson et al., "Tensional homeostasis and the malignant phenotype," Cancer Cell, vol. 8, no. 3, pp. 241-254, 2005.

[15] J. A. Hubbell, "Biomaterials in tissue engineering," Nature Biotechnology, vol. 13, no. 6, pp. 565-576, 1995.

[16] M. J. Bissell, D. C. Radisky, A. Rizki, V. M. Weaver, and O. W. Petersen, "The organizing principle: microenvironmental influences in the normal and malignant breast," Differentiation, vol. 70, no. 9-10, pp. 537-546, 2002.

[17] J.-W. Shin and D. J. Mooney, "Extracellular matrix stiffness causes systematic variations in proliferation and chemosensitivity in myeloid leukemias," Proceedings of the National 
Academy of Sciences of the United States of America, vol. 113, no. 43, pp. 12126-12131, 2016.

[18] J. Werfel, S. Krause, A. G. Bischof et al., "How changes in extracellular matrix mechanics and gene expression variability might combine to drive cancer progression," PLoS One, vol. 8, no. 10, p. e76122, 2013.

[19] A. P. Sarvazyan, A. R. Skovaroda, S. Y. Emelianov et al., "Biophysical bases of elasticity imaging," Acoustical Imaging, vol. 21, pp. 223-240, 1995.

[20] C. Sumi, K. Nakayama, and M. Kubota, "An effective ultrasonic strain measurement-based shear modulus reconstruction technique for superficial tissues-demonstration on in vitro pork ribs and in vivo human breast tissues," Physics in Medicine \& Biology, vol. 45, no. 6, pp. 1511-1520, 2000.

[21] P. P. Provenzano, D. R. Inman, K. W. Eliceiri et al., "Collagen density promotes mammary tumor initiation and progression," BMC Medicine, vol. 6, no. 1, p. 11, 2008.

[22] J. Debnath, S. K. Muthuswamy, and J. S. Brugge, "Morphogenesis and oncogenesis of MCF-10A mammary epithelial acini grown in three-dimensional basement membrane cultures," Methods, vol. 30, no. 3, pp. 256-268, 2003.

[23] C. S. Hughes, L. M. Postovit, and G. A. Lajoie, "Matrigel: a complex protein mixture required for optimal growth of cell culture," Proteomics, vol. 10, no. 9, pp. 1886-1890, 2010.

[24] C. S. Szot, C. F. Buchanan, J. W. Freeman, and M. N. Rylander, " $3 \mathrm{D}$ in vitro bioengineered tumors based on collagen I hydrogels," Biomaterials, vol. 32, no. 31, pp. 7905-7912, 2011.

[25] K. H. Bouhadir, E. Alsberg, and D. J. Mooney, "Hydrogels for combination delivery of antineoplastic agents," Biomaterials, vol. 22, no. 19, pp. 2625-2633, 2001.

[26] A. M. Kloxin, C. J. Kloxin, C. N. Bowman, and K. S. Anseth, "Mechanical properties of cellularly responsive hydrogels and their experimental determination," Advanced Materials, vol. 22, no. 31, pp. 3484-3494, 2010.

[27] R. J. Pelham and Y. L. Wang, "Cell locomotion and focal adhesions are regulated by substrate flexibility," Proceedings of the National Academy of Sciences of the United States of America, vol. 94, no. 25, pp. 13661-13665, 1997.

[28] A. J. Engler, S. Sen, H. L. Sweeney, and D. E. Discher, "Matrix elasticity directs stem cell lineage specification," Cell, vol. 126, no. 4, pp. 677-689, 2006.

[29] T. Yeung, P. C. Georges, L. A. Flanagan et al., "Effects of substrate stiffness on cell morphology, cytoskeletal structure, and adhesion," Cell Motility and the Cytoskeleton, vol. 60, no. 1, pp. 24-34, 2005.

[30] S. C. Wei, L. Fattet, J. H. Tsai et al., "Matrix stiffness drives epithelial-mesenchymal transition and tumour metastasis through a TWIST1-G3BP2 mechanotransduction pathway," Nature Cell Biology, vol. 17, no. 5, pp. 678-688, 2015.

[31] C. M. Williams, A. J. Engler, R. D. Slone, L. L. Galante, and J. E. Schwarzbauer, "Fibronectin expression modulates mammary epithelial cell proliferation during acinar differentiation," Cancer Research, vol. 68, no. 9, pp. 3185-3192, 2008.

[32] S. Zustiak, R. Nossal, and D. L. Sackett, "Multiwell stiffness assay for the study of cell responsiveness to cytotoxic drugs," Biotechnology and Bioengineering, vol. 111, no. 2, pp. 396-403, 2014.

[33] J. K. Mouw, G. Ou, and V. M. Weaver, "Extracellular matrix assembly: a multiscale deconstruction," Nature Reviews Molecular Cell Biology, vol. 15, no. 12, pp. 771-785, 2014.

[34] P. P. Provenzano, K. W. Eliceiri, J. M. Campbell, D. R. Inman, J. G. White, and P. J. Keely, "Collagen reorganization at the tumor-stromal interface facilitates local invasion," $B M C$ Medicine, vol. 4, no. 1, p. 38, 2006.

[35] D. O. Croci, M. F. Zacarías Fluck, M. J. Rico, P. Matar, G. A. Rabinovich, and O. G. Scharovsky, "Dynamic crosstalk between tumor and immune cells in orchestrating the immunosuppressive network at the tumor microenvironment," Cancer Immunology, Immunotherapy, vol. 56, no. 11, pp. 1687-1700, 2007.

[36] R. M. Desai, S. T. Koshy, S. A. Hilderbrand, D. J. Mooney, and N. S. Joshi, "Versatile click alginate hydrogels crosslinked via tetrazine-norbornene chemistry," Biomaterials, vol. 50, pp. 30-37, 2015.

[37] J. Zhu, "Bioactive modification of poly(ethylene glycol) hydrogels for tissue engineering," Biomaterials, vol. 31, no. 17, pp. 4639-4656, 2010.

[38] K. M. Mabry, R. L. Lawrence, and K. S. Anseth, "Dynamic stiffening of poly(ethylene glycol)-based hydrogels to direct valvular interstitial cell phenotype in a three-dimensional environment," Biomaterials, vol. 49, pp. 47-56, 2015.

[39] M. Guvendiren and J. A. Burdick, "Stiffening hydrogels to probe short- and long-term cellular responses to dynamic mechanics," Nature Communications, vol. 3, p. 792, 2012.

[40] A. M. Rosales, K. M. Mabry, E. M. Nehls, and K. S. Anseth, "Photoresponsive elastic properties of azobenzene-containing poly(ethylene-glycol)-based hydrogels," Biomacromolecules, vol. 16, no. 3, pp. 798-806, 2015.

[41] R. S. Stowers, S. C. Allen, and L. J. Suggs, "Dynamic phototuning of 3D hydrogel stiffness," Proceedings of the National Academy of Sciences of the United States of America, vol. 112, no. 7, pp. 1953-1958, 2015.

[42] R. S. Stowers, S. C. Allen, K. Sanchez et al., "Extracellular matrix stiffening induces a malignant phenotypic transition in breast epithelial cells," Cellular and Molecular Bioengineering, vol. 10, no. 1, pp. 114-123, 2017.

[43] S. R. Sershen, S. L. Westcott, N. J. Halas, and J. L. West, "Temperature-sensitive polymer-nanoshell composites for photothermally modulated drug delivery," Journal of Biomedical Materials Research, vol. 51, no. 3, pp. 293-298, 2000.

[44] M. Hu, J. Chen, Z.-Y. Li et al., "Gold nanostructures: engineering their plasmonic properties for biomedical applications," Chemical Society Reviews, vol. 35, no. 11, pp. 1084-1094, 2006.

[45] S. Eustis and M. A. el-Sayed, "Why gold nanoparticles are more precious than pretty gold: noble metal surface plasmon resonance and its enhancement of the radiative and nonradiative properties of nanocrystals of different shapes," Chemical Society Reviews, vol. 35, no. 3, pp. 209-217, 2006.

[46] L. E. Strong, S. N. Dahotre, and J. L. West, "Hydrogelnanoparticle composites for optically modulated cancer therapeutic delivery," Journal of Controlled Release, vol. 178, pp. 63-68, 2014.

[47] Y. Qu, B. Y. Chu, J. R. Peng et al., “A biodegradable thermoresponsive hybrid hydrogel: therapeutic applications in preventing the post-operative recurrence of breast cancer," NPG Asia Materials, vol. 7, no. 8, p. e207, 2015.

[48] J. Song, S. Hwang, K. Im et al., "Light-responsible DNA hydrogel-gold nanoparticle assembly for synergistic cancer therapy," Journal of Materials Chemistry B, vol. 3, no. 8, pp. 1537-1543, 2015.

[49] Y. Ye and X. Hu, "A pH-sensitive injectable nanoparticle composite hydrogel for anticancer drug delivery," Journal of Nanomaterials, vol. 2016, Article ID 9816461, 8 pages, 2016. 
[50] C. Nowald, B. T. Käsdorf, and O. Lieleg, "Controlled nanoparticle release from a hydrogel by DNA-mediated particle disaggregation," Journal of Controlled Release, vol. 246, pp. 71-78, 2017

[51] N. Segovia, M. Pont, N. Oliva, V. Ramos, S. Borrós, and N. Artzi, "Hydrogel doped with nanoparticles for local sustained release of siRNA in breast cancer," Advanced Healthcare Materials, vol. 4, no. 2, pp. 271-280, 2015.

[52] S. B. Campbell, M. Patenaude, and T. Hoare, "Injectable superparamagnets: highly elastic and degradable poly $(\mathrm{N}-$ isopropylacrylamide)-superparamagnetic iron oxide nanoparticle (SPION) composite hydrogels," Biomacromolecules, vol. 14, no. 3, pp. 644-653, 2013.

[53] O. B. Ayyub and P. Kofinas, "Enzyme induced stiffening of nanoparticle-hydrogel composites with structural color," ACS Nano, vol. 9, no. 8, pp. 8004-8011, 2015.

[54] S. K. Maji, S. Sreejith, A. K. Mandal, X. Ma, and Y. Zhao, "Immobilizing gold nanoparticles in mesoporous silica covered reduced graphene oxide: a hybrid material for cancer cell detection through hydrogen peroxide sensing," ACS Applied Materials \& Interfaces, vol. 6, no. 16, pp. 13648-13656, 2014. 

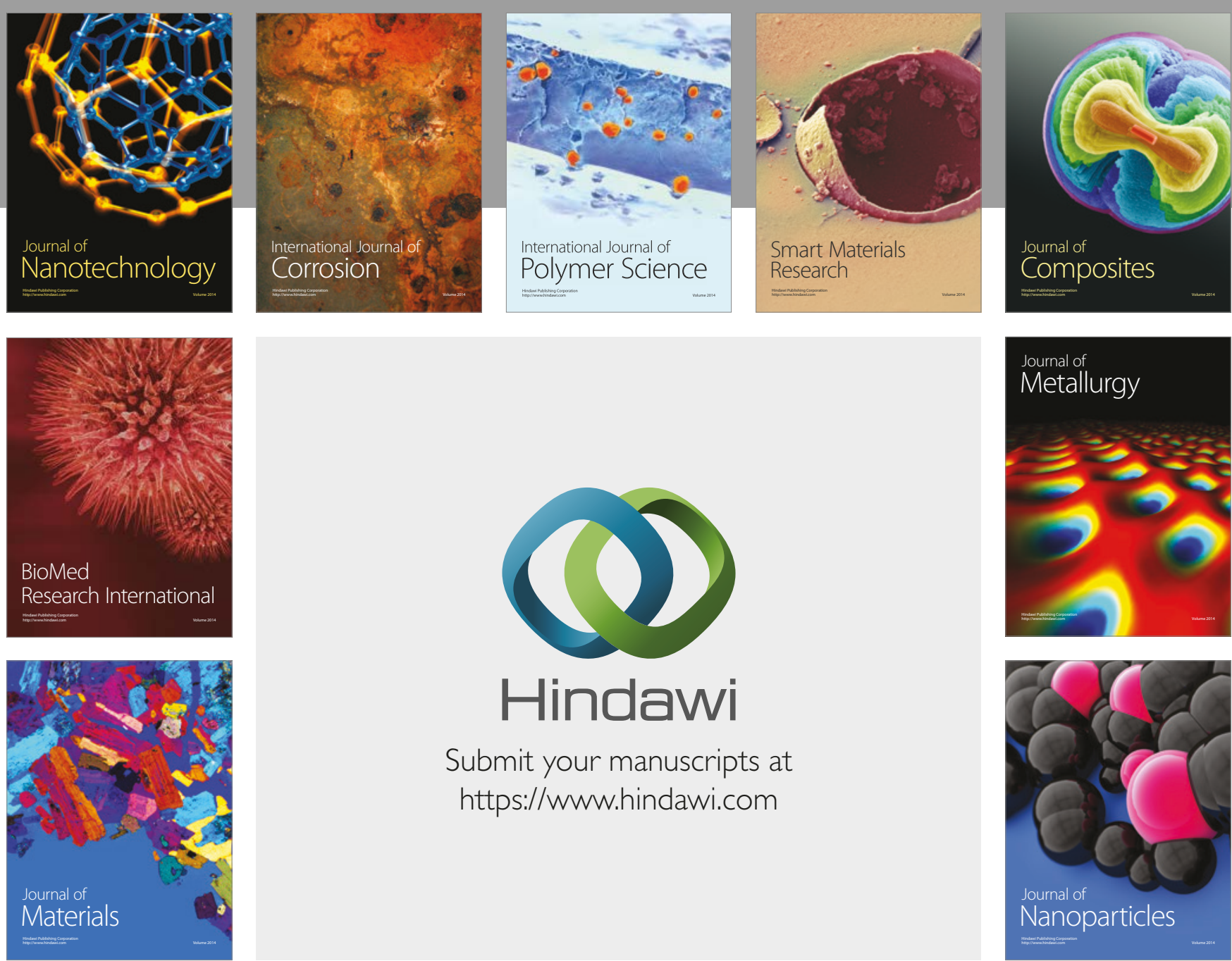

\section{Hindawi}

Submit your manuscripts at

https://www.hindawi.com
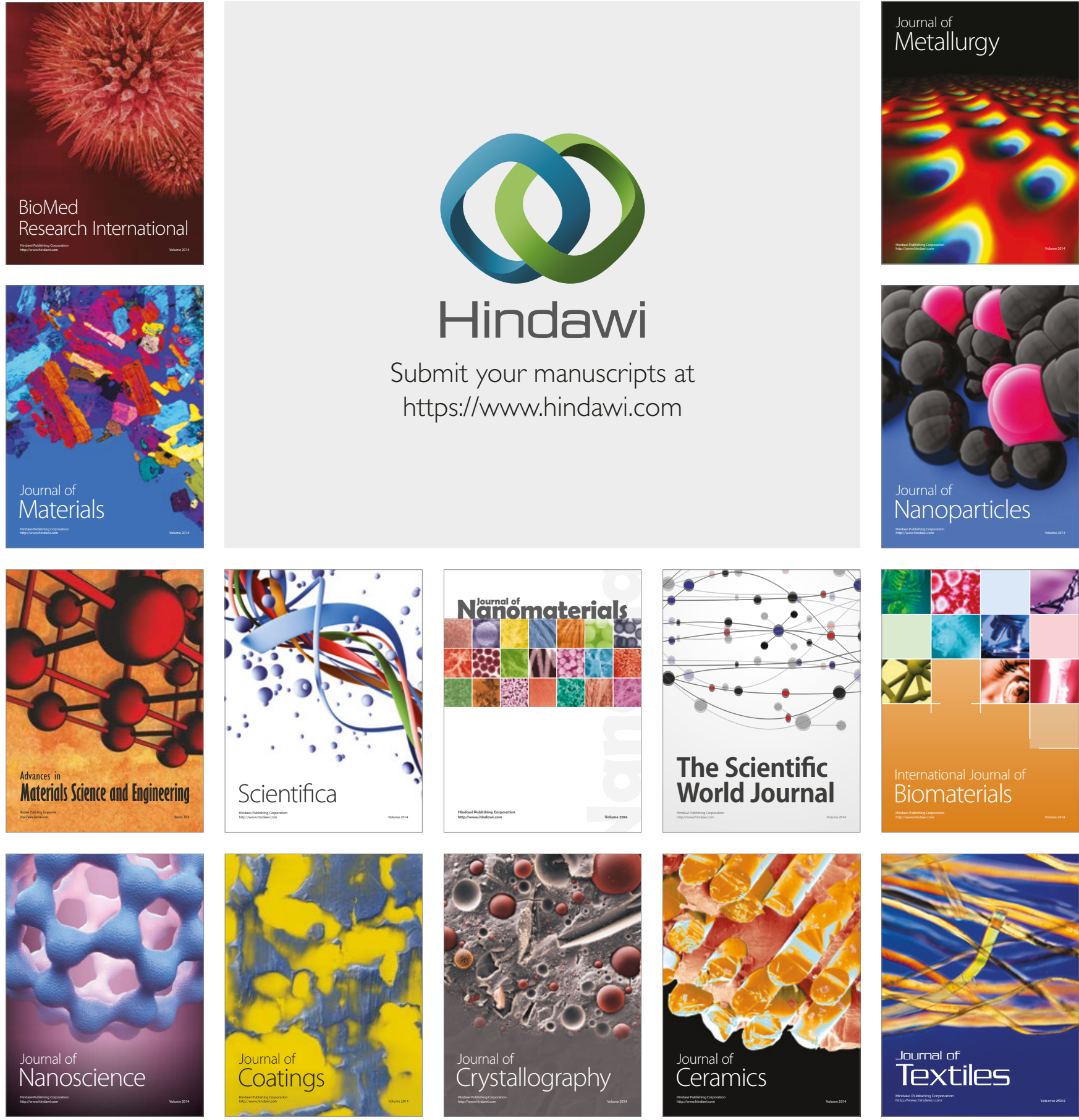

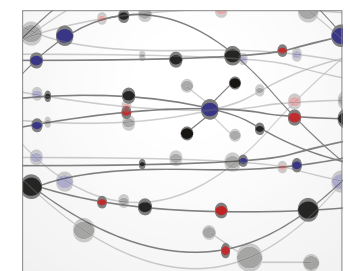

The Scientific World Journal
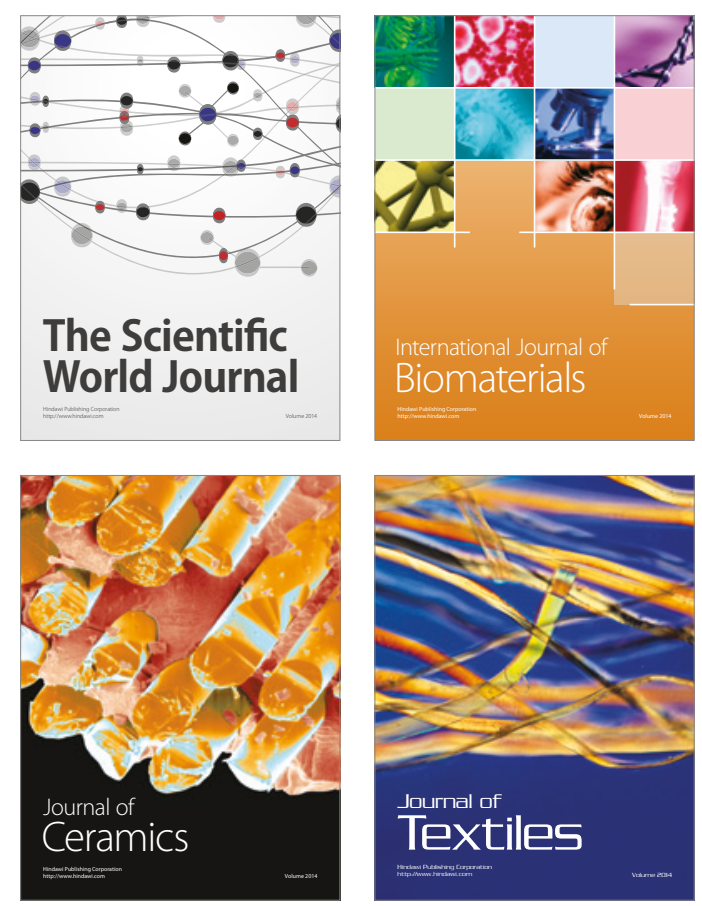